\title{
ANÁLISIS DE LA NUEVA LEY DE URGENCIA MÉDICA PARA LA DETECCIÓN OPORTUNA Y ATENCIÓN INTEGRAL DEL CÁNCER DEL NIÑO Y ADOLESCENTE
}

\author{
ANALYSIS OF THE NEW MEDICAL EMERGENCY LAW FOR THE TIMELY \\ DETECTION AND INTEGRAL CARE OF CHILD AND ADOLESCENT CANCER
}

Ursula Bazán Dobbertin*

Investigadora independiente

Lima-Perú

https: / / orcid.org/0000-0002-6599-8612

ursula@bazanfantinato.com

\section{Resumen}

El presente artículo analiza el texto de la Ley 31041 - Ley de Urgencia Médica para la Detección Oportuna y Atención Integral del Cáncer del Niño y Adolescente, aprobada por el Congreso de la República el 31 de julio de 2020 y publicada el 02 de setiembre del mismo año. Si bien la norma constituye un aporte necesario para brindar protección a la población infantil y adolescente diagnosticada con cáncer, hubiera sido oportuno que se contemplen diversos principios de protección a los integrantes de la familia.

Palabras clave: Cáncer, protección a los integrantes de la familia, Interés Superior del Niño, enfoque de género, discapacidad, adultos mayores.

\section{Abstract:}

This article analyzes the text the Law of Medical Emergency for the Timely Detection and Comprehensive Care of Child Cancer, approved by the Congress of the Republic on July 31, 2020 and published on September 2 of the same year. Although this law constitutes a necessary contribution to provide protection to the child and adolescent population diagnosed with cancer, it would have been opportune to contemplate various principles of protection for family members.

Keywords: Cancer, Child's Best Interest, gender approach, disability, older adults.

Candidata a Magíster en Derecho Civil con mención en Derecho de Familia por la Universidad Femenina del Sagrado Corazón (UNIFE). Abogada titulada por la Pontificia Universidad Católica del Perú. Socia fundadora del Estudio Bazán \& Fantinato Abogados. 


\section{INTRODUCCIÓN}

El 31 de julio de 2020 fue aprobada por el Congreso de la República la Ley de Urgencia Médica para la Detección Oportuna y Atención Integral del Cáncer del Niño y Adolescente, con la finalidad de que se garantice la atención integral de calidad para niños, niñas y adolescentes con cáncer. Sin embargo, aunque la iniciativa es importante para abordar esta problemática, existen aspectos concernientes a los principios de protección de las familias y sus integrantes que no han sido contemplados.

\section{LOS NUEVOS PARADIGMAS DEL DERECHO DE FAMILIA EN EL CONTEXTO DEL CÁNCER INFANTIL}

La recientemente aprobada Ley de Urgencia Médica para la Detección Oportuna y Atención Integral del Cáncer del Niño y Adolescente establece que su objeto es "la detección oportuna y atención integral de calidad de los niños y adolescentes con enfermedades oncológicas"; sin embargo, aparentemente no se ha considerado que un gran número de pacientes no reside en Lima y se verá obligado a salir de sus hogares para poder acceder al tratamiento.

“Un 70\% de pacientes menores de 15 años que atiende el INEN son de provincias" (Fernández, 2018, s/p), lo que implica que ese porcentaje de pacientes junto con las personas que los acompañan, dejan de ser parte de la vida cotidiana que transcurre en su hogar y muchas veces no cuentan con un lugar en donde albergarse, a diferencia de los que tienen sus hogares en la capital.

El cáncer altera la dinámica familiar, una vez recibido el temible diagnóstico, el o la paciente deberá trasladarse en forma regular a las consultas médicas y someterse a una serie de procedimientos, los tratamientos producirán estragos en quien los recibe y necesitará ser cuidado o cuidada por algún miembro de su familia. Ello cobra mayor complejidad cuando se trata de personas que viven en provincia y requieren seguir su tratamiento en Lima; si a esto le sumamos la variable etaria, cuando el paciente es niño, niña o adolescente, este traslado se producirá con la compañía de uno de sus progenitores o algún adulto responsable de su cuidado, quien dejará de realizar las actividades que usualmente cumplía en el hogar; produciéndose así fenómenos migratorios en busca de atención médica de calidad. 
Lo señalado líneas arriba concuerda con lo indicado por Jelin (2007) cuando señala que "Los procesos migratorios siempre implican la fragmentación de las unidades familiares, sea de manera temporaria o más permanente" (p.112), en tal sentido, las familias que viven fuera de la capital se desfragmentarán al tener que migrar cuando uno de sus integrantes es diagnosticado con cáncer.

Como bien indica Jelin (2007), en ocasiones existe una confusión entre familia y hogar cuando lo cierto es que "las responsabilidades y obligaciones familiares pueden estar a cargo de miembros que no comparten el hogar ( $p$. 96)", lo cual claramente va a ocurrir una vez que se produzca la migración en búsqueda del tratamiento médico, pues la persona afectada con la enfermedad y su acompañante van a salir de su lugar de origen, mientras que el resto de la familia permanecerá en el hogar. En estos casos, los y las pacientes no solo van a verse privados de su salud, sino también de la interacción cotidiana con los miembros de su familia, aspectos que merecían ser tomados en cuenta.

Jelin (2007) señala que "la mayor parte de los movimientos migratorios -sean temporarios o permanentes, internos o internacionales- tienen una motivación económica que combina factores de expulsión y de atracción" (pp.112). Si aplicamos lo señalado por la autora a la migración de niños, niñas o adolescentes con cáncer (junto con su o sus acompañantes) podemos colegir lo siguiente: que este fenómeno es un movimiento migratorio interno, en tanto existe un traslado dentro del país (hacia Lima); que existen factores de expulsión, los cuales son la enfermedad en sí y la falta de condiciones para poder llevar el tratamiento en sus lugares de origen y; factores de atracción, que serían los hospitales, la atención y la infraestructura que existe en Lima para poder atender a la o el paciente.

Las migraciones producidas a raíz del cáncer infantil están motivadas por la búsqueda de un tratamiento adecuado para combatir la enfermedad y, si bien no están determinadas por la clásica búsqueda de oportunidades laborales y mejoras económicas, podemos señalar que encajan con la idea de que "Lo que resulta significativo es que las responsabilidades se desligan de la convivencia y la interacción cotidianas" (Jelin, 2007, p.114); lo cual va a ocurrir debido a que las personas que se trasladarán a Lima para conseguir el tratamiento médico, dejarán atrás (al menos temporalmente) la convivencia y la dinámica diaria.

"Lo correcto, en términos de protección internacional, es referirse a las familias, en plural (Ramírez, 2018, p.322)” y, siguiendo la línea de Jelin 
(2007), respecto a que familia no es sinónimo de hogar, podemos señalar que era preciso proteger a aquellos individuos que, aunque siguen siendo parte de la misma familia, no compartirán un mismo techo.

Esta pluralidad a que se refiere Ramírez (2018), debe extenderse también a aquellos casos en que el niño, niña o adolescente no se encuentra bajo los cuidados de sus progenitores, lo cual tampoco ha sido contemplado por la norma pues al momento de establecer una licencia de un año con goce de haber, se considera únicamente a los trabajadores con hijos menores de 18 años diagnosticados con cáncer. Nos explicamos: la primera disposición complementaria modificatoria agregó un párrafo al artículo 2 de la Ley 30421 (Ley que concede el derecho de licencia a trabajadores con familiares directos que se encuentran con enfermedad grave o terminal o sufran de algún accidente). En dicho párrafo se establece una licencia con goce de haber no mayor a un año para el trabajador que tenga un hijo niño o adolescente menor de 18 años con cáncer, sin contemplar la posibilidad de que este niño, niña o adolescente no se encuentre bajo los cuidados de sus progenitores sino de otros familiares como, por ejemplo, los abuelos que requerirán de esta licencia para poder lidiar con la enfermedad.

El Tribunal Constitucional peruano, en la sentencia recaída en el expediente Nro. 01643-2014-PA/TC, estableció "la familia, al ser un instituto natural, se encuentra inevitablemente a merced de los nuevos contextos sociales" y que, fenómenos tales como la migración y la participación de las mujeres en el mercado laboral, han motivado que sus estructuras sean distintas a la tradicional nuclear y estén compuestas por padres, hijos, abuelos y tíos. El Tribunal Constitucional señala que en estas familias, cuando los padres están imposibilitados por razones laborales o de otra índole, serán los abuelos quienes supervisen las actividades de sus nietos. Lo señalado por el Tribunal Constitucional también puede ser aplicado al contexto de cáncer en pacientes menores de 18 años, pues esta licencia alude a trabajadores formales cuando señala que esta licencia deberá ser cubierta "los primeros veintiún días por el empleador y el tiempo restante por EsSalud"; lo que quiere decir que los hijos de trabajadores por cuenta propia que estén a cargo de otros familiares (por ejemplo abuelos) que sí cuenten con empleos formales, no podrán beneficiarse de esta licencia.

La Corte Interamericana de Derechos Humanos en el Caso Ramírez Escobary otros Vs. Guatemala señaló que "el Estado debe respetar y garantizar la plenitud de los derechos consagrados en la Convención, así como algunas medidas especiales para su adecuada supervivencia y desarrollo"; a ello podemos agregar que, parte de estas medidas especiales, es adecuar las leyes 
a los diversos tipos de familia de las que niños, niñas y adolescentes pueden ser integrantes, por lo que es fundamental que una ley de esta naturaleza alcance a los diversos tipos de organización familiar.

En esa línea Acosta (2007) asevera que "los Estados tienen la obligación de brindar la colaboración que los núcleos familiares requieren para proveer el en entorno protector que los seres humanos necesitan. (p.209)", por lo que cualquier medida legislativa o política pública que pretenda establecerse debe ir de la mano con la realidad social y, en lo que a familias se refiere, deben tener como horizonte la protección de sus integrantes independientemente del tipo de organización familiar a la que pertenezcan. En materia de cáncer ello reviste de gran importancia, pues las personas diagnosticadas van a requerir de una protección reforzada por parte del Estado, lo que no vemos reflejado en la ley bajo análisis, en tanto deja sin la atención de una licencia con goce de haber a los niños, niñas y adolescentes que no están a cargo de sus padres. Por lo tanto, un problema que se desprende de la norma es que las políticas que busca implementar, no son acordes con el Plan Nacional de Fortalecimiento a las Familias 2016-2021, el cual explícitamente establece que el Estado deberá implementar políticas para que las familias (sea cual fuere su origen u organización) puedan cumplir con sus funciones, indicando que estas se traducen en el "cuidado, seguridad económica y afecto entre sus miembros. (pp. 19)" y que, en caso de enfermedades oncológicas, se requiere de un mayor apoyo por parte del Estado para su cumplimiento.

\section{LA NECESIDAD DE CONTEMPLAR EN LA NORMA AL NIÑO, NIÑA Y ADOLESCENTE COMO VERDADERO SUJETO DE PROTECCIÓN}

De acuerdo con la Observación General Nro. 14 del Comité de los Derechos del Niño, uno de los elementos que debe tomarse en cuenta para evaluar el Interés Superior del Niño es su derecho a la salud (fundamento 77); en ese sentido, podemos indicar que la ley aprobada por el Congreso de la República reconoce la potencial vulnerabilidad de los niños, niñas y adolescentes, estableciendo una serie de disposiciones para la detección oportuna y tratamiento integral del cáncer. Sin embargo, hemos podido observar que la norma no acoge dicho principio en forma integral y no contempla en plenitud al niño, niña y adolescente como sujeto de protección, ello desde que hace alusión en algunos artículos al término "menor" (propio de la Doctrina de la Situación Irregular) y porque no hace mención a su derecho a ser oído, al no destinar artículo alguno a su capacidad de decisión para aceptar o no el someterse a determinados tratamientos. 
Dicha Observación General, establece que el derecho a la salud del niño, niña y adolescente implica que se le brinde la información necesaria respecto de los riesgos y beneficios de los tratamientos a los que se les vaya a someter $\mathrm{y}$, de ser posible, ofrecerle la oportunidad de dar su consentimiento (fundamento 77); no obstante, ello, la ley bajo análisis no hace referencia alguna a ello.

De otro lado, aunque se establece una política de protección para la niñez y adolescencia, ha asumido lo que Picontó (2016) denomina una "concepción homogénea de la infancia", que considera que "la auténtica infancia se relaciona con la vida familiar, doméstica, en la que niños y niñas son cuidados por los adultos, olvidando a las 'otras infancias' que son aquellas que no viven en el seno de una familia (p.145)". Señalamos esto porque la licencia con goce de haber que establece la norma para los padres de niños, niñas o adolescentes con cáncer (Primera Disposición Complementaria Modificatoria) parte de la premisa de que estos últimos viven dentro de una familia en la que uno de sus padres es trabajador formal; sin embargo, la ley bajo comentario deja de lado a esas "otras infancias" a que se refiere Picontó, que en nuestro país están representadas por los niños en situación de calle, niños institucionalizados en albergues y a los privados de la libertad, entre otros.

\section{LA AUSENCIA DE UN ENFOQUE DE GÉNERO EN LA NORMA}

Como señalábamos al inicio de este documento, cuando un miembro de la familia es diagnosticado con cáncer, la dinámica de la familia cambia máxime si se trata de un niño, niña y adolescente, pues va a requerir del acompañamiento de alguno de sus padres o adultos responsables para trasladarse con la finalidad de recibir los tratamientos que necesita. Tomando en consideración que, pese a que el mercado laboral cuenta con una mayor participación femenina, las labores del hogar se les siguen designando a las mujeres (Jelin, 2007), lo que va a implicar que en la mayoría de casos sea la madre (u otra mujer responsable) quien acompañe al paciente a las consultas médicas y a los procedimientos.

Este acompañamiento implica una salida de la mujer de la privacidad del hogar, de ese lugar interno desde donde estaba acostumbrada a socializar, para introducirse en la esfera pública en donde va a recurrir al Estado para realizar una serie de trámites para que su familiar afectado con cáncer pueda ser tratado. Este fenómeno es los que Rábago (2008) denomina "del patio a la plaza", en donde "el patio representa un espacio interno de socialización que 
se crea desde el hogar (p.16)". Por su parte Zined, citado por Rábago (2008), señala que la plaza sería "un lugar de emancipación y de liberación (p.16)".

Ocurre que, al momento en que un niño, niña o adolescente tiene un diagnóstico de cáncer, su madre o acompañante dejará de lado su espacio de socialización y entrará en interacción con el Estado para solicitar o exigir el tratamiento integral que ofrece la norma.

Además de salir del hogar, la mujer deberá también dejar de lado sus actividades económicas, pues existirá una gran demanda de tiempo para que pueda realizar el acompañamiento a las consultas y procedimientos médicos, lo que implicará que no pueda generar ingresos para su familia. En ese sentido consideramos que, si bien la norma ha considerado una licencia para el trabajador que tenga un hijo menor de dieciocho años diagnosticado con cáncer, esa protección únicamente está prevista para los empleos formales dejando de lado al empleo no formal. Esto reviste mayor dificultad cuando la mujer no cuenta con un empleo formal pues no habrá una licencia que la ampare, debiendo depender económicamente de su pareja u otros familiares mientras acompaña a su hijo o hija para que siga su tratamiento.

En efecto, una proporción de mujeres bastante significativa en América Latina trabaja por cuenta propia en diversas actividades, de las cuales, un gran número pertenece al sector informal, lo que implica una escaza protección social, en el caso específico de nuestro país, las estadísticas de Cepal del año 2014 indican que el 38.8\% de mujeres trabaja por cuenta propia y el 8.5.\% se dedica al trabajo doméstico (Ullman, Maldonado y Nieves: 2014), lo que implica que ellas no se van a beneficiar con la licencia que establece la norma; en tal sentido la mujer que pertenece a este grupo, en cada oportunidad en que tenga que acompañar al paciente con cáncer para que pueda someterse a sus tratamientos médicos, se verá obligada a dejar de percibir ingresos, sin licencia que la atienda.

De otro lado, esta situación se agrava cuando nos encontramos frente a familias con jefatura femenina, cuyo número viene aumentado en América Latina. Como señalan Ullmann, Maldonado y Nieves (2014) “al interior de los países, los estratos bajos (hogares en pobreza, del primer quintil y /o vulnerables a la pobreza) presentan mayores niveles de dependencia, mayor presencia de monoparentalidad con jefatura femenina y mayor número de personas en promedio (p. 55)" y; en caso de las mujeres que trabajan dentro de la economía informal, la percepción de menores ingresos no solo afectará a la propia mujer y al paciente, sino también a todos sus dependientes más aún si se trata de hogares monoparentales en que la mujer es el único sustento de sus familiares. 
En el año 2014, el porcentaje de hogares monoparentales encabezados por mujeres ascendían al 12,4\% y su frecuencia es mayor en los llamados "sectores pobres" que en los "sectores no pobres" (Ullmann, Maldonado y Nieves, 2014); lo que nos muestra que la política de licencias no corresponde a las estadísticas de nuestra población, pues no solo se está obviando a las mujeres que trabajan en el sector informal, sino que también está descuidado aquellas cuyos ingresos muchas veces son ínfimos. Por ejemplo, si es diagnosticado con cáncer uno de los hijos de una madre soltera dedicada al trabajo doméstico "independiente" (con ingresos por día de trabajo), ella no solo estará fuera del ámbito de la licencia, sino que dejará de percibir entradas por cada día en que no acuda a laborar. Ello demuestra que, además de la ausencia de un enfoque de género, no se ha tomado en cuenta la data respecto a la evolución de las familias del Perú, en donde las mujeres son la cabeza y muchas veces la única fuente de ingresos y de cuidado.

Mencionamos el tema del cuidado pues, el problema no solo lo constituye el tema de la licencia, sino que un rasgo en común que tienen las mujeres independientemente de su tipo de hogar, es que en la mayoría de ellas recae la responsabilidad del cuidado de los niños; al respecto Ullman, Martínez y Nieves (2014) señalan que "existe una fuerte rigidez en la división sexual del trabajo dentro del hogar y las mujeres siguen siendo las principales proveedoras de cuidado infantil y en general de los miembros dependientes (p.50)", ¿qué quiere decir ello?, que la mujer no solo va a carecer de la licencia, sino que igualmente no se ha previsto de qué forma se la va a reemplazar en las labores de atención y cuidado de sus dependientes cada vez que tenga que acudir con el paciente a sus consultas médicas y tratamientos; lo cual confirma lo asentado por Arriagada (2006) respecto a que la sobrevaloración de la idea de que las mujeres son quienes se encargan de la familia, hace necesario el establecimiento de políticas que las apoyen en el ejercicio de sus funciones pues "el costo de oportunidad del trabajo destinado al cuidado familiar es cada vez mayor. (p.23)", costo de oportunidad que se incrementará exponencialmente si a sus "rutinarias" tareas de cuidado se le agrega la responsabilidad de atender a un hijo o hija con cáncer con todo lo que ello implica.

La licencia que establece la norma constituye lo que Blofield y Martínez (2014) denominan política secuencial, que en buena cuenta se trata de "medidas que protegen la seguridad de los ingresos durante los tiempos —mensuales, semanales o diarios- destinados a los cuidados. (pp. 109)". Dichas autoras indican que la "secuenciación" puede durar meses y colocan como ejemplo a las licencias de maternidad. En el caso materia de análisis la secuenciación se está estableciendo por 12 meses y "el cuidado permanece en 
las familias, históricamente en las mujeres (Blofield y Martínez, 2014, p.109)"; por lo tanto, se parte de la premisa de que es la familia la responsable de las labores del cuidado y, si bien no establece distinción entre padres y madres, es generalmente la mujer quien se va a ocupar de ello. Asimismo, se trata de una política que Goldani, citado por Arriagada (2006) llama "política referida a la familia", pues tiene como finalidad el reforzamiento de las funciones de esta, en este caso la atención y el cuidado de uno de sus miembros que con diagnóstico de cáncer.

La norma señala que la licencia con goce de haber será otorgada de manera "excepcional y única de acuerdo con las necesidades del trabajador cuyo hijo, niño o adolescente menor de 18 años sea diagnosticado con cáncer (Primera Disposición Complementaria Modificatoria)", pero quedan a la interpretación dos aspectos: el primero referente a qué debería entenderse por excepcional, pues no existe ninguna precisión al respecto y; el segundo, a si la referencia al trabajador (en singular) respecta a ambos progenitores o a uno de ellos. Si se interpreta que son ambos progenitores a quienes se les va a otorgar la licencia, podríamos señalar que se trata de una política de corresponsabilidad paterna para "balancear la presencia de los padres en los cuidados (Blofield y Martínez, 2014, p. 111)", lo que permitiría que el o la paciente con cáncer cuente con la compañía de su padre y su madre para poder acudir a los tratamientos o que, al menos, este acompañamiento sea alternado entre ambos; sin embargo, si la norma se refiere a solo uno de los dos progenitores, seguiríamos enmarcados dentro del paradigma de las mujeres como cuidadoras.

\section{LA NECESIDAD DE UNA POLÍTICA QUE PERMITA LA CONCILIACIÓN ENTRE LA VIDA LABORAL Y FAMILIAR}

Efectivamente, uno de los lineamientos del Plan Nacional de Fortalecimiento a las Familias 2016 - 2021 (2016) es el "Fomento de las responsabilidades compartidas y de la conciliación entre la vida familiar y el trabajo", sin embargo, de acuerdo a lo explicado podemos señalar que la ley aprobada por el Congreso de la República no se condice con este lineamiento, no solo por las características que debe tener el trabajador a quien se otorgará la licencia (empleo formal), sino también porque no se establece qué políticas se van a establecer pasado el año de la licencia y qué mecanismos aplicar para que la o el trabajador pueda realizar sus actividades laborales sin dejar de lado su dedicación a la familia.

En cuanto a la duración de la licencia, la norma es clara cuando señala que no será mayor a un año, lo que no siempre va a responder 
a las necesidades del o la paciente con diagnóstico de cáncer, porque, de prolongarse el tratamiento a un período mayor, quedaría un vacío respecto a qué políticas se van a establecer para que el niño, niña o adolescente pueda ser acompañado para la continuación del mismo, pasado el año. En ese orden de ideas, hubiera sido importante que se establezca una política que no solo permita conciliar la vida familiar con la vida laboral, sino que además concilie esos aspectos con el acompañamiento y el cuidado de la persona con cáncer. Como Sunkel (2007) señala, es trascendental que las políticas permitan resolver el conflicto del trabajo con la familia pues "permiten aumentar el tiempo disponible para la familia (padres o madres) de los trabajadores/as, cuestión central en el conflicto familia - trabajo. (p.182)"; el autor ejemplifica ello con las jornadas laborales a tiempo parcial, los horarios especiales que permitan que el trabajo se realice en las noches y los permisos familiares como "permiso maternal, permiso paternal y otros permisos por razones familiares (por ejemplo, disposiciones en el caso de que un hijo esté enfermo) (p. 183)". En esa línea, pasado el año que dura la licencia, quedaría en el aire lo concerniente a los cuidados del o la paciente y demás dependientes en caso que el tratamiento requiera de un período mayor, por lo cual sería indispensable que se implementen este tipo de políticas en que se permita la adaptación de las jornadas laborales para que el o la trabajadora pueda distribuir sus horarios de acuerdo a las necesidades de su familia o que, por ejemplo, se establezca permisos para que pueda realizar las labores de cuidado y/o acompañamiento a la persona enferma.

\section{LA FALTA DE IMPACTO DE LA NORMA EN MATERIA DE DISCAPACIDAD}

Es un hecho conocido que, al menos temporalmente, el cáncer va a generar alguna discapacidad en quien lo padece: los efectos secundarios de las quimioterapias, la pérdida de algún órgano o extremidad van a constituir factores que podrían limitar a la persona; sin embargo, no se ha contemplado alguna política con la que ello se pueda enfrentar, como tampoco se hace referencia a la especial vulnerabilidad de los niños, niñas o adolescentes que tenían una discapacidad preexistente al diagnóstico de cáncer; lo cual acarrearía mayores dificultades que las propias de la enfermedad en sí.

La discapacidad está representada por tres modelos: el modelo de la prescindencia, el modelo rehabilitador y el modelo social. El modelo de la prescindencia, que consideraba que la discapacidad tenía un origen religioso señalando que se debía prescindir de las personas con discapacidad; el modelo rehabilitador, que establecía que la discapacidad era un problema o déficit de la persona, señalando que era necesario ocultar o "curar" la discapacidad 
para que la persona pueda integrarse a la sociedad y; el modelo social que propugna que la discapacidad no está en el individuo per se, sino más bien en la sociedad, la cual coloca trabas para su integración, este modelo tiene una mirada basada en un enfoque de derechos humanos señalando que la sociedad debe garantizar que la persona con discapacidad pueda gozar y ejercer sus derechos en igualdad de condiciones con los demás (Palacios, 2015).

Lo señalado por la autora resulta interesante para este análisis en tanto se advierte que no se parte de un modelo social de discapacidad, centrándose en un modelo rehabilitador por cuanto abarca la enfermedad desde su diagnóstico hasta la finalización del tratamiento, pero no se hace mención a la atención de las necesidades de las personas para su desenvolvimiento en los diferentes aspectos de su vida. Consideramos que pudo mejorarse el enfoque, estableciendo medidas que garanticen el ejercicio pleno de los derechos de los niños, niñas y adolescentes con cáncer en igualdad de condiciones que los demás, tales como el derecho a la educación, en donde se podría establecer la obligación de los centros educativos de tomar las medidas pertinentes para que el niño diagnosticado con cáncer pueda culminar el año escolar sin interrumpir su tratamiento (clases virtuales, adecuación de currícula, etc.).

A propósito del derecho a la educación, la Observación General Nro. 6 sobre la igualdad y la no discriminación del Comité de los Derechos de las Personas con Discapacidad (2018), señala que los Estados Partes tienen la obligación de "asegurar una educación inclusiva a las personas con discapacidad en igualdad de condiciones con las demás, en la comunidad en que viven." (fundamento 64), lo que se puede conseguir mediante ajustes razonables tales como adaptar los sistemas de evaluación, el material didáctico y las estrategias de enseñanza (fundamento 23).

La Observación General Nro. 6, responde a un modelo social de discapacidad que podría ser acogido por la ley materia de análisis, en la cual hubiera sido óptimo incluir los aspectos concernientes a las adaptaciones que deberían realizar los centros educativos para que el niño, niña o adolescente con cáncer no vea interrumpidos sus estudios; por ejemplo, brindarles fechas y formas de evaluación distintas a las estandarizadas, facilitar que sus aulas estén ubicadas en el primer piso o establecer programas de aulas hospitalarias, entre otros. 


\section{LOS PRINCIPIOS CONSTITUCIONALES DE PROTECCIÓN A LA FAMILIA DESCUIDADOS: LAS PERSONAS ADULTAS MAYORES}

Huenchuan (2018), citando a Williams, señala que la infancia y las personas mayores tienen en común la dependencia, pero la diferencia entre ambos grupos radica en que "Mientras que en la infancia las personas son protegidas para que logren desarrollar la capacidad de ser autónomas, en la vejez, por el contrario, son despojadas de la posibilidad de decidir por sí mismas a medida que su edad avanza (p.88)."; podemos señalar entonces que la infancia y adolescencia son consideradas etapas de la vida con una proyección a futuro para contribuir a la sociedad, mientras que se asume a la vejez como una etapa en que va menguando la autonomía y, con ello, las personas van perdiendo su productividad; motivo por el cual también requieren de una protección específica y, sin embargo, no han sido siquiera mencionados en la norma.

Los principios constitucionales de protección a la familia se derivan del artículo $4^{\circ}$ de nuestra Carta Magna, uno de ellos es el de "protección especial de la niñez, adolescencia, maternidad y ancianidad" (Ramírez, 2020). Hemos observado que la norma, cuando menos intenta proteger a la niñez, sin embargo, se ha descuidado un importante grupo de población vulnerable, el cual está constituido por las personas adultas mayores y, con ello, se ha dejado de lado el principio de protección a la ancianidad.

Nuestra Constitución Política establece en su artículo 4 el principio de protección de la ancianidad (Ramírez, 2020) y, por su parte, la Ley del Adulto Mayor (Ley 28803), reconoce que "La persona adulta mayor es sujeto prioritario de la atención integral en materia de salud, así como en los casos de emergencia médica o en los que se presenten enfermedades de carácter terminal (artículo 9)"; sin embargo, no existe disposición que asegure su atención oportuna y prioritaria en caso de padecer cáncer en cualquiera de sus etapas. Vemos que, en cuanto a adultos mayores, se hace énfasis en las emergencias médicas y en las enfermedades de carácter terminal, cuando no siempre el cáncer va a ser detectado en esta última etapa.

De otro lado, la Convención Interamericana Sobre la Protección de los Derechos Humanos de las Personas Mayores lleva cinco años de aprobada sin haber sido, a la fecha, ratificada por nuestro país. Dicha convención contiene una serie de principios y definiciones que requieren ser adoptados en nuestras leyes, como lo es el de "Envejecimiento activo y saludable" que se describe de la siguiente forma: 
Proceso por el cual se optimizan las oportunidades de bienestar físico, mental y social, de participar en actividades sociales, económicas, culturales, espirituales y cívicas, y de contar con protección seguridad y atención, con el objetivo de ampliar la esperanza de vida saludable y la calidad de vida de todos los individuos en la vejez, y permitirles así seguir contribuyendo activamente a sus familias, amigos, comunidades y naciones.

Este concepto de envejecimiento saludable resulta fundamental para poder incluir a las personas adultas mayores dentro de una ley como la que se está analizando pues, para garantizar una vida saludable y de calidad, es necesario que se trate oportunamente cualquier enfermedad que es detectada, incluida el cáncer.

El Plan Nacional de Fortalecimiento a las Familias 2016-2021 (2016), señala que la población de personas que superan los 60 años de edad ha venido en aumento de acuerdo con el censo del año 2007 y; como bien señala Rossel (2016), en América Latina se está desarrollando un proceso de envejecimiento de la población referido a un crecimiento demográfico de las personas adultas mayores que, para el año 2025 en países como el nuestro este grupo va a conformar "el 15\% del total de la población (p.36)". De acuerdo con la autora, ello va a significar "nuevas demandas a los sistemas de salud, en especial de apoyos y servicios formales de cuidado para personas adultas mayores, así como de enfermos crónicos. (p.37).". Este aumento de la población adulta mayor no solo va a exigir que se establezcan políticas que permitan que los sistemas de salud abarquen a este grupo, sino también que se satisfaga la demanda de cuidado a que se refiere Rossel (2016), máxime si se encuentra de por medio el diagnóstico de una enfermedad oncológica, lo cual constituye parte de la agenda que no ha incluido la norma.

\section{CONCLUSIONES}

1. La ley de urgencia médica para la detección oportuna y atención integral del cáncer del niño y adolescente que aprobó el Congreso de la República, no ha contemplado la especial vulnerabilidad en que se encuentran los pacientes con cáncer que se ven obligados a migrar a Lima desde las provincias del Perú y que no tienen donde albergarse.

2. Si bien se ha establecido una licencia de un año con goce de haber para los trabajadores con hijos menores de 18 años diagnosticados con cáncer, no contempla aquellos casos en que los o las pacientes están a cargo de otros familiares (por ejemplo, los abuelos), que van a requerir de dicha licencia. 
3. Al no contemplar a los y las pacientes a cargo de familiares distintos a sus padres, podemos indicar que esta ley no es acorde con el Plan Nacional de Fortalecimiento a las Familias 2016-2021, pues este señala que las políticas deben contribuir a que la familia pueda cumplir con sus funciones independientemente de su origen y organización.

4. Si bien esta ley tiene por objeto garantizar la atención integral de niños, niñas y adolescentes con cáncer, no acoge en plenitud el principio del Interés Superior del Niño en tanto no establece aspectos concernientes al ejercicio de su derecho a ser oídos respecto a la posibilidad de brindar su consentimiento para someterse a determinados tratamientos.

5. Se ha partido de una concepción "homogénea de la infancia", pues las licencias que establece corresponden a pacientes que viven en el seno de una familia, descuidando a las denominadas "otras infancias" que no están bajo el cuidado de adultos.

6. Sería necesario que se incluya un enfoque de género en donde brinde especial protección a la mujer que se ocupa del cuidado cotidiano de la persona diagnosticada con cáncer.

7. La licencia con goce de haber que se ha establecido constituye una política secuencial para garantizar los ingresos del trabajador o trabajadora que tengan un hijo o hija con diagnóstico de cáncer.

8. Si se buscara adaptar la ley bajo análisis a los modelos con que se representa a la discapacidad, se encontraría dentro del modelo rehabilitador en tanto aborda a la enfermedad y su tratamiento, pero no establece aspectos concernientes al ejercicio pleno de los derechos de la persona diagnosticada con cáncer.

9. Se ha dejado de lado el principio de protección constitucional de la ancianidad, pues no se ha incluido a los adultos mayores quienes constituyen también población cuya proporción viene aumentando y es potencialmente vulnerable y requiere protección especial. 


\section{REFERENCIAS}

Acosta, G. (2007). Cambios legislativos en la formación y disolución de familias: una mirada de contexto. Familias y políticas públicas en América Latina: Una historia de desencuentros. Santiago de Chile: Comisión Económica para América Latina y el Caribe (CEPAL), 201-210.

Arriagada, I. (2006). Cambios en las políticas sociales: políticas de género y familia. Santiago de Chile: CEPAL, 2006, pp. 1-23.

Blofield, M. y Martínez, J. (2014). Trabajo, familia y cambios en la política pública en América Latina: equidad, maternalismo y corresponsabilidad. Revista CEPAL, 114, 107-125

Comité de los Derechos del Niño (2013). Observación general N. 14 sobre el derecho del niño a que su interés superior sea una consideración primordial (artículo 3, párrafo 1).

Comité sobre los Derechos de las Personas con Discapacidad (2018). Observación general N. ${ }^{\circ} 6$ sobre la igualdad y la no discriminación.

Corte Interamericana de Derechos Humanos. Caso Ramírez Escobar y otros Vs. Guatemala. Fondo, Reparaciones y Costas. Resumen oficial de la sentencia de 9 de marzo de 2018. Serie C No. 351

Fernández, Cristina (15 de febrero de 2018). Cáncer infantil: más de 1600 casos se registran al año en Perú. Diario El Comerio Sección Sucesos https: / / elcomercio.pe/lima/sucesos/cancer-infantil-1600-casos-registran-ano-peru-noticia-497395-noticia /

Huenchuan, S. (2018). Los derechos humanos en un contexto de envejecimiento y la protección de los derechos de las personas mayores. Envejecimiento, personas mayores y Agenda 2030 para el Desarrollo Sostenible: perspectiva regional y de derechos humanos. Santiago: Comisión Económica para América Latina y el Caribe (CEPAL), 85-134.

Jelin, E. (2007). Las familias latinoamericanas en el marco de las transformaciones globales. Familias y políticas públicas en América Latina: Una historia de desencuentros. Santiago de Chile: CEPAL, 2007, 93-124 
Ministerio de la Mujer y Poblaciones Vulnerables. Plan Nacional de Fortalecimiento a las Familias 2016 - 2021.

Olsen, F. (1999). El mito de la intervención del Estado en la familia. FACIO, Alda y Lorena FRIES. Género y derecho. Santiago: LOM Ediciones, 541- 567.

Organización de Estados Americanos (2015). Convención interamericana sobre la protección de los derechos humanos de las personas mayores.

Palacios, A. (2015). Una introducción al modelo social de la discapacidad y su reflejo en la Convención sobre los derechos de las personas con discapacidad". Nueve conceptos claves para entender la Convención sobre los derechos de las personas con discapacidad. Lima: IDEHPUCP, 2015, 933.

Picontó, T. (2016). Fisuras en la protección de los derechos de la infancia. Cuadernos Electrónicos de Filosofía del Derecho, 33, 133-166.

Rábago, M. (2008). Los conceptos de esfera pública y privada en el Derecho de Familia. Mujeres, familia y trabajo. México D.F. 1-26.

Ramírez, B. (2018). Las familias desde los estándares de la jurisprudencia interamericana: una mirada comparada de la legislación argentina y peruana. Revista De Familia, Revista interdisciplinaria de Doctrina y Jurisprudencia, 84, 2018, 317 - 339

Ramírez, B. (mayo, 2020). Protección constitucional de la familia: Curso de Políticas Públicas. Universidad Femenina del Sagrado de Corazón.

Rossel, C. (2016). Desafíos demográficos para la organización social del cuidado y las políticas públicas. Santiago de Chile: CEPAL, 3564 .

Sunkel, G. (2007). Regímenes de bienestar y políticas de familia en América Latina. Familias y políticas públicas en América Latina: Una historia de desencuentros. Santiago de Chile: Comisión Económica para América Latina y el Caribe (CEPAL), 171-186. 
Tribunal Constitucional del Perú (2018). Sentencia del de 14 de agosto. Expediente 01643-2014-PA/TC.

Ullmann, H.; Maldonado, C. Valera y Nieves, I (2014). La evolución de las estructuras familiares en América Latina, 1990-2010. Los retos de la pobreza, la vulnerabilidad y el cuidado. Santiago de Chile: CEPAL. https: / / elcomercio.pe/lima/sucesos / cancer-infantil-1-600casos-registran-ano-peru-noticia-497395-noticia/?ref=ecr

Fecha de recepción: 11-09-2020

Fecha de aceptación: 26-10-2020 